\title{
Alternately repetitive cast/brace (ARCB) treatment for larger-magnitude early-onset scoliosis: A retrospective cohort study
}

\author{
Kawakami N*, Saito T, Tauchi R, Kawakami K and Ohara T \\ Department of Orthopedics and Spine Surgery, Meijo Hospital, Nagoya, Japan
}

\begin{abstract}
Objective: The present study aims to investigate the potential of ARCB-T as a delayed tactic even for larger-magnitude scoliosis.

Methods: In the present retrospective cohort study, the inclusion criteria were as follows: (1) EOS and 2) age of initiation for ARCB-T $\leq 4$ years. Consecutively enrolled 120 patients since 1995-2016 met these criteria. They were divided into the following two groups in terms of initial scoliosis of 50 ${ }^{\circ}$ : Cast Larger-Group (CL-G; main scoliosis $\geq 50^{\circ} ; n=78$ ) and Cast Mild-Group (CM-G; main scoliosis $<50^{\circ} ; n=42$ ). Etiologies were as follows: congenital/structural defects (CS/ST; $n$ $=55$ ); infantile idiopathic scoliosis (IIS; $n=28$ ); syndromic scoliosis (SS; $n=32$ ); and neuromuscular scoliosis $(n=5)$. ARCB-T was switched to surgical intervention in 52 and 17 patients in the CL-G and CM-G, respectively. The endpoints were the progression rate during ARCB-T and the magnitude of scoliosis at the end of ARCB-T. We compared scoliosis measured at initiation and end of ARCB-T, and scoliosis in the initial cast placement between the two groups.

Results: We observed a reduction of scoliosis $<30^{\circ}$ in $11.5 \%$ and $28.6 \%$ patients in the CL-G and CM-G, respectively. While, patients with scoliosis $>70^{\circ}$ did not display improvement of scoliosis $<30^{\circ}$. Early initiation of ARCB-T significantly correlated with a lower progression rate $(P=0.0384)$. Patients with larger-magnitude scoliosis at the first casting exhibited significantly larger-magnitude scoliosis at the end of ARCB-T $(P<0.0001)$. Better correction of scoliosis by initial casting decreased the progression rate $(P=0.0113)$ among patients in both the groups. Although the correction of scoliosis by initial casting did not correlate with the progression rate in the CL-G $(P=0.1153)$, the progression rate during ARCB-T significantly correlated with the correction by casting in patients with $\geq 70^{\circ}$ or $80^{\circ}$ of scoliosis $(P=0.0016)$. The diagnoses correlated with the efficacy of ARCB-T and IIS exhibited a significantly better progression rate $\left(-3.0^{\circ} /\right.$ year $)$ than other etiologies.

Conclusion: Despite being limited in the suppression of the progression of larger-magnitude scoliosis, ARCB-T works less efficiently as a delayed tactic to surgery and could be an option for larger-magnitude scoliosis if it displays better correction at the first cast placement.
\end{abstract}

\section{Introduction}

Alternately repetitive cast/brace treatment (ARCB-T) is performed to decrease scoliosis and/or delay the need for surgery for mild-to-moderate early-onset scoliosis (EOS). However, limited studies have investigated the clinical outcomes of patients with largermagnitude scoliosis.

In the last two decades, studies of surgical outcomes of growthfriendly surgery for early-onset scoliosis (EOS) have demonstrated that a too early initiation of surgical intervention often leads to poor outcomes because of multiple surgical complications, as well as unpredicted spontaneous fusion [1-5]. Since Mehta [6] reintroduced serial cast treatment for EOS with cases of some patients exhibiting improvement like most cured state, cast placement for EOS has returned as an effective treatment option in routine clinical practice to decrease scoliosis and as a delayed tactic before surgical treatment.

Sanders [7] reported their series of patients treated with serial cast placement with two aims. The first aim was to decrease the magnitude of scoliosis. The author reported that EOS patients with smaller curvature $\left[\geq 50^{\circ}-60^{\circ}\right.$, single curvature, less rotation, infantile idiopathic scoliosis (IIS)] were more likely to exhibit the reduction of scoliosis through serial cast treatment. Moreover, the study emphasized the efficacy of initiating serial cast treatment at age $<2$ years. The second aim was a delayed tactic for surgical intervention. Besides IIS, many patients with non-idiopathic scoliosis often visit scoliosis clinic at the age $>2-3$ years complaining of moderate-severe curvatures; however, this might be too late or not expected to attain the reduction of scoliosis by applying serial cast treatment.

To date, several studies have reported the efficacy of serial cast treatment, even for older EOS patients with non-idiopathic/syndromic larger curvature, to delay the need for surgery [7-12]. Some of those studies enrolled EOS patients with the curvature $>80^{\circ}[7,9,10,13]$. Nevertheless, to the best of our knowledge, no study has detailed the efficacy of serial cast treatment for such a severe curvature. Moreover, limitations and complications of serial cast treatment remain partially elucidated.

In the present study, we hypothesize that serial cast application is effective even for larger curvatures in terms of a delayed tactic. Hence,

*Correspondence to: Noriaki Kawakami, Department of Orthopaedics and Spine Surgery, Meijo Hospital, 1-3-1, Sannomaru, Naka-ku, Nagoya, 460-0001, Japan, E-mail: nupriver@gmail.or.jp

Key words: serial cast treatment, early onset scoliosis (eos), alternately repetitive cast/brace (arcb) treatment, corrective brace

Received: October 23, 2019; Accepted: November 03, 2019; Published: November 07, 2019 
this study aims to assess the efficacy and limitations of serial cast treatment for larger curvatures in EOS as a delayed tactic for surgical treatment.

\section{Characteristics of our serial cast treatment combined with brace application and the timing of switching to surgical intervention}

We have been performing cast application for EOS since 1995. In the first several years, our candidates for cast treatment were young pediatric patients with severe scoliosis. Since 2000, we extended cast application to almost all patients with EOS $35^{\circ}-40^{\circ}$, except congenital scoliosis. We applied serial cast placement for patients with scoliosis $<35^{\circ}$ if patients have congenital scoliosis on the lumbar spine for prevention of secondary wedging of adjacent normal vertebrae. Of note, all cast applications were conducted on a Risser Table (Matsumoto Prosthetics \& Orthotics Manufacturing Co. Ltd.) with correction by traction and derotation with straps. At the time of cast application, general anesthesia was not used. A corrective cast was routinely applied for 2-3 weeks followed by wearing of a corrective brace (CTSLO for thoracic curvatures and TLSO for thoracolumbar or lumbar curvatures) for several weeks. The brace-wearing duration was dependent on the magnitude, type, and etiology of scoliosis. The cast placement was reapplied if we established that brace treatment could not maintain scoliosis with acceptable magnitude. Owing to this repeat cycle of cast and brace applications, we termed our cast and brace treatment as "alternately repetitive cast/brace treatment" (ARCB-T) [14]. Typically, ARCB-T was performed until the age of nearly 7 years; this was the time when the school years began, and almost all patients no longer wanted cast placement. At this point, ARCB-T was switched to pure brace treatment if patients were still required to continue wearing a brace.

The timing of when to switch to a surgical intervention, such as growth-friendly surgery or short fusion based on the curve magnitude and etiology, was ascertained by the following findings: (1) uncontrollable progression during ARCB-T was determined at the age $>6$ years; (2) collapsed chest cage deformity was induced due to cast application even wihtout progression of scoliosis, (3) when the type of curvature or etiology (most were congenital scoliosis) could be treated with short fusion (fusion $\leq 6$ segments); or (4) combination with thoracolumbar or lumbar angular kyphosis.

\section{Methods}

\section{Study design and study cohort}

In this retrospective cohort study, the inclusion criteria were as follows: (1) EOS; 2) age of initiation for ARCBT-T $\leq 4$ years. Consecutively enrolled 120 patients since 1995-2016 met the above criteria. These 120 patients were divided into two groups: Cast MildGroup (CM-G; main scoliosis $<50^{\circ}$ ) and Cast Larger-Group (CL-G; main scoliosis $\geq 50^{\circ}$ ) in terms of $50^{\circ}$ of initial main curvature.

The CL-G included 78 patients ( 24 males, 54 females; mean age: 2.9 years at the first casting). The CM-G included 42 patients (21 males, 21 females; mean age: 2.5 years at the first casting). Etiologies among patients in both the groups included congenital/structural defects (CS/ST; $n=55$ ); IIS; $n=28$ ); syndromic scoliosis (SS; $n=32$ ); and neuromuscular scoliosis (NM; $n=5$ ), based on the 2014 EOS classification [15].

Scoliosis just before the first cast application, in the first cast placement, and at the time of the final follow-up or just at the end of
ARCB-T were measured by two authors, including one who applied the casts to patients. For data comparison and to investigate the efficacy of ARCB-T for a larger curvature, we compared the CL-G with the CM-G.

\section{Endpoints}

In the present study, the endpoints were the progression rate during ARCB-T and the magnitude of scoliosis at the end of ARCB-T. The final objective was to determine the efficacy of ARCB-T in patients with larger-magnitude scoliosis compared with smaller-magnitude scoliosis by comparing these endpoints.

\section{Statistical analysis}

All statistical analyses were performed with jump vision 13.0 (Cary, $\mathrm{NY}$ ). We used the nonparametric Wilcoxon rank-sum test to investigate the difference of continuous measures within or across the groups. Furthermore, all $p<0.05$ were considered statistically significant.

\section{Results}

Between both the groups, we observed significant differences in sex, age at the first cast placement, and etiology; however, followup time and the number of cast placements during ARCB-T did not differ (Table 1, Figure 1). Surgical intervention was executed following ARCB-T in 52 patients (66.7\%) in the CL-G, with the age and duration from the first cast placement to surgery of $7.1 \pm 2.3$ years and $3.8 \pm$ 2.1 (range: $0.3-8.8$ ) years, respectively. In the CM-G; however, surgical intervention was executed in $17(40.5 \%)$ patients, with the age and duration from the first cast placement to surgery of $6.1 \pm 1.5$ years and $3.4 \pm 1.6$ (range: 1.3-8.9) years, respectively. In addition, the percentage of surgical intervention exhibited a significant difference between both the groups $(P=0.00070)$, although no significant difference was noted in age at surgery and duration from the first cast placement to surgery.

Scoliosis measured at all intervals showed significant differences between the two groups $(P<0.0001)$. However, the progression and initial correction rate by cast placement did not exhibit any significant difference between both the groups (Table 2).

Table 1. Demographic data of two groups $\left({ }^{*} \mathrm{p}=0.0379,{ }^{* *} \mathrm{p}=0.0211\right)$

\begin{tabular}{|c|c|c|}
\hline & Cast-Mild Group (CM-G) & $\begin{array}{c}\text { Cast Larger-Group } \\
\text { (CL-G) }\end{array}$ \\
\hline Number pf participants & 42 & 78 \\
\hline Sex & Male 21, Female $21^{*}$ & Male 24, Female 54* \\
\hline Age at $1^{\text {st }}$ visit $/ 1^{\text {st }}$ Cast (y) & $2.5 \pm 0.9^{* *}$ & $2.9 \pm 1.1^{* *}$ \\
\hline FU (y) & $4.8 \pm 2.9$ & $4.3 \pm 2.7$ \\
\hline Total cast count & $5.4 \pm 3.9$ & $6.9 \pm 4.4$ \\
\hline
\end{tabular}
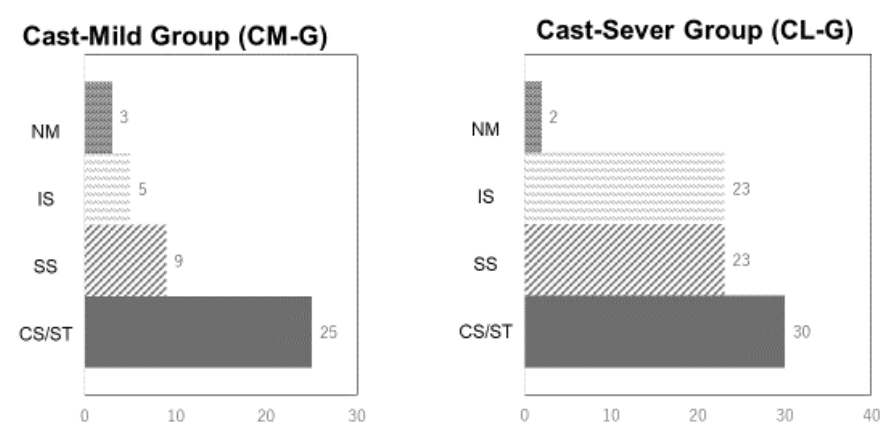

Figure 1. Etiology in two groups (Patients were categorized into four categories, such as congenital/structural (CS/ST), infantile idiopathic scoliosis, syndromic scoliosis, and neuromuscular scoliosis based on the early-onset scoliosis classification. CS/ST was the majority in both Cast Mild-Group and Cast Larger-Group. There was a significant difference between the two groups $(P=0.0402)$ 
Initial scoliosis just before the first cast placement exhibited a positive correlation with the magnitude of scoliosis at the end of ARCB-T or the final follow-up time (Figure 2); however, it did not exhibit any significant correlation between initial scoliosis and progression rate $(/ y)$. The early initiation of ARCB-T significantly correlated with a lower progression rate $(P=0.0330)$. The later initiation of ARCB-T caused a higher magnitude of scoliosis $(P=$ $0.0001)$. In addition, etiology affected the magnitude of scoliosis at the end of ARCB-T and the progression rate during ARCB-T. Patients with IIS exhibited the lowest magnitude of scoliosis at the end of ARCB-T in the four etiologies (Table 3). Furthermore, the progression rate was also lowest in IIS of all etiologies. We observed no significant difference in the progression rate between the CM-G and CL-G across all etiologies (Table 4).

The improvement of scoliosis $<30^{\circ}$ at the end of ARCB-T was observed in $12(28.6 \%)$ and nine patients $(11.5 \%)$ of the CM-G and CL-G, respectively. We noted a significant difference in the percentage of scoliosis improvement during ARCB-T between both the groups $(P=0.0159)$. This tendency was also observed when all patients were re-categorized into two groups by the magnitude of initial scoliosis of $60^{\circ}$ or $70^{\circ}$ (Figure 3). No patients in the larger group divided by $>70^{\circ}$ showed improvement.

IIS exhibited a much higher percentage of scoliosis improvement (35.7\%) in all etiologies $(P=0.0366$; Figure 4). Furthermore, four patients $(80 \%)$ with IIS in the CM-G exhibited improvement to scoliosis $<30^{\circ}$, whereas six patients $(26.0 \%)$ with IIS in the CL-G exhibited improvement $(P=0.0228)$.

The initial correction (\%) at the first cast placement as a whole showed a weakly negative correlation with the progression rate $(\% / y)$ of scoliosis during ARCB-T $\left(\mathrm{R}^{2}=0.053586, P=0.0113\right)$. The evaluation of this correlation in each group revealed that the initial correction did not significantly correlate with the progression rate in the CL-G

Table 2. Scoliosis between two groups

\begin{tabular}{|c|c|c|c|}
\hline & $\begin{array}{c}\text { Cast Mild-Group } \\
\text { (CM-G) }\end{array}$ & $\begin{array}{c}\text { Cast Larger-Group } \\
\text { (CL-G) }\end{array}$ & p value \\
\hline Number pf patients & 42 & 78 & \\
\hline Initial scoliosis $\left(^{\circ}\right)$ & $40 \pm 7(25-49)$ & $65 \pm 14(50-114)$ & $<0.0001$ \\
\hline In-cast scoliosis $\left(^{\circ}\right)$ & $20 \pm 10(1-45)$ & $35 \pm 14(7-68)$ & $<0.0001$ \\
\hline $\begin{array}{c}\text { Scoliosis at the end of } \\
\text { conservative Tx. }\left(^{\circ}\right)\end{array}$ & $41 \pm 18(11-79)$ & $64 \pm 26(8-115)$ & $<0.0001$ \\
\hline Progression $\left({ }^{\circ} \mathbf{y}\right)$ & $0.6 \pm 4.5$ & $02 \pm 6.3$ & 0.5914 \\
\hline Initial correction rate $(\%)$ & $50 \pm 23$ & $46 \pm 18$ & 0.4353 \\
\hline
\end{tabular}

Table 3. Diagnoses correlated to the effectiveness of ARCB-T

\begin{tabular}{|c|c|c|c|c|cc|}
\hline & CS/ST & IS & NM & SS & \\
\hline $\mathrm{N}$ & 55 & 28 & 5 & 32 & \\
\hline $\begin{array}{c}\text { Scoliosis at } \\
\text { the end of } \\
\text { ARCB-T }\end{array}$ & $57 \pm 23$ & $43 \pm 25$ & $59 \pm 20$ & $67 \pm 29$ & $\begin{array}{l}\text { IS vs SS } 0.0013 \\
\text { IS vs CS/ST } 0.0178\end{array}$ \\
\hline $\begin{array}{c}\text { Progression } \\
\text { rate }\end{array}$ & $0.7 \pm 5.4$ & $-3.0 \pm 4.4$ & $5.1 \pm 5.5$ & $2.7 \pm 7.6$ & $\begin{array}{l}\text { IS vs SS } 0.0007 \\
\text { IS vs CS/ST } 0.0007 \\
\text { IS vs NM }\end{array} 0.0129$ \\
\hline
\end{tabular}

Table 4. Progression during ARCB-T between two groups in terms etiology

\begin{tabular}{|c|c|c|c|c|}
\hline & CS/ST & IS & NM & SS \\
\hline CM-G & $1.1 \pm 6.3$ & $-3.5 \pm 4.6$ & $5.0 \pm 3.5$ & $3.2 \pm 8.3$ \\
\hline CL-G & $0.3 \pm 4.1$ & $-1.1 \pm 2.7$ & $5.2 \pm 7.4$ & $0.9 \pm 5.2$ \\
\hline p-value & 0.7609 & 0.6101 & --- & 0.5274 \\
\hline
\end{tabular}

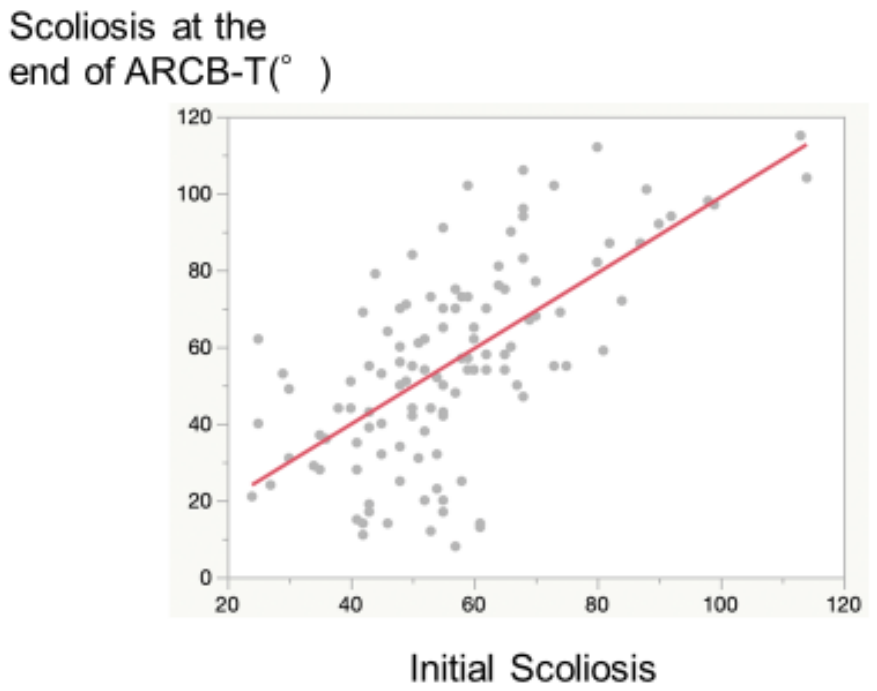

Figure 2. Relation between Initial Scoliosis and Scoliosis at the End of ARCB-T. The correlation between initial scoliosis and scoliosis at the end of alternately repetitive cast/ brace treatment (ARCB-T). A positive correlated existed between scoliosis at the end of ARCB-T $(\square)$ and initial scoliosis just before ARCB-T $(R 2=0.422831, P<0.0001)$

(a)

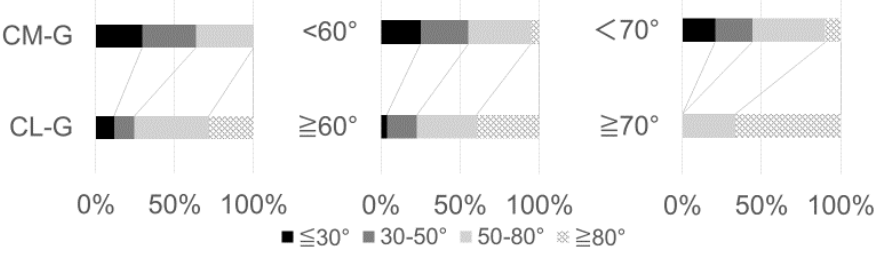

Figure 3. Percentage of Improvement in Scoliosis at the End of ARCB-T (The percentage of patients who exhibited the improvement of scoliosis by alternately repetitive cast/brace treatment (ARCB-T) in three patterns of classification in terms of the magnitude of initial scoliosis before ARCB-T. a) Groups of patients classified by $50^{\circ}$ of initial scoliosis before ARCB-T $\left(<50^{\circ}: 28.6 \%\right.$ vs. $\left.\geq 50^{\circ}: 11.5 \%, P=0.00186\right)$. b) Groups of patients classified by $60^{\circ}$ of initial scoliosis before ARCB-T $\left(<60^{\circ}: 24.1 \%\right.$ vs. $\left.\geq 60^{\circ}: 4.9 \%, P=0.0081\right)$. c) Groups of patients classified by $70^{\circ}$ of initial scoliosis before ARCB-T $\left(<70^{\circ}: 20.1 \%\right.$ vs. $\geq 70^{\circ}: 0 \%, P=0.0330$ ). The percentage of patients who exhibited improvement of scoliosis $<30^{\circ}$ at the end of ARCB-T tends to decrease in groups of patients with larger and larger scoliosis before ARCB-T)

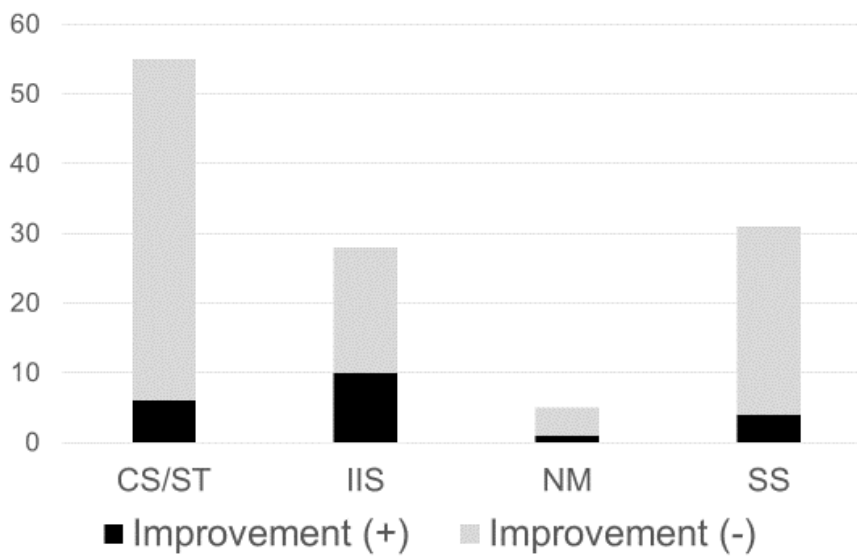

Figure 4. Improvement of Scoliosis in Etiologies (Patients with infantile idiopathic scoliosis exhibited the highest percentage of improvement of scoliosis by alternately repetitive cast/ brace treatment $(P=0.0366)$ 
$\left(\mathrm{R}^{2}=0.032308, P=0.1153\right)$. Conversely, the $\mathrm{CM}-\mathrm{G}$ exhibited a stronger negative correlation between the initial correction and progression rate $\left(\mathrm{R}^{2}=0.192627, P=0.0041\right.$; Figure 5).

Besides CM-G and CL-G, when patients were also re-categorized into two groups in terms of the magnitude of scoliosis of $60^{\circ}, 70^{\circ}$, or 80 , both groups with scoliosis $\geq 70^{\circ}$ and $\geq 80^{\circ}$ clearly exhibited significantly negative correlations between the progression rate and the initial correction, but not in the group with scoliosis $\geq 60^{\circ}$ (Figure 6).

In all patients, surgical interventions could be delayed for $47 \pm 35$ months by using ARCB-T. No significant difference in the duration of delay for surgery was noted between the CM-G and the CL-G. If all patients were re-categorized by magnitude of initial scoliosis of $60^{\circ}, 70^{\circ}$, or $80^{\circ}$, surgical intervention in patients with scoliosis $>80^{\circ}$ could be significantly delayed for only 2319 months; the duration was clearly shorter than others (Figure 7).

Besides minor complications, such as skin sloughing and decubitus, which did not affect the strategy of ARCB-T, major complications caused by cast placement occurred in five patients in the CL-G and in none in the CM-G. Of the five patients in the CL-G, three experienced progressive windswept chest cage deformities; the chest cage slowly deformed following the progression of scoliosis during ARCB-T. One patient with Marfan syndrome, who had a funnel chest, had a history of surgical treatment using the NUSS method; although cast application was cautiously performed by opening bilateral two windows on the lateral wall of the cast to avoid pressure sore in ARCB-T, the patient developed deep decubitus on the lateral chest wall because of hardware prominence that required the removal of chest bars. The fifth patient a) All patients

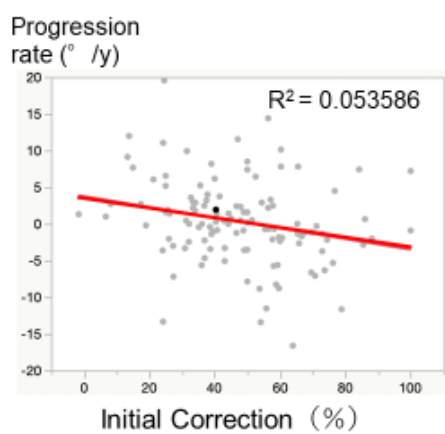

b) CL-G

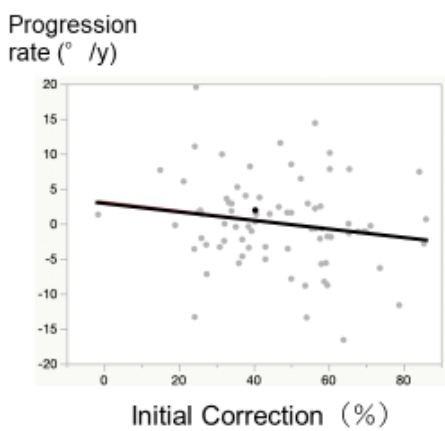

c) $\mathrm{CM}-\mathrm{G}$

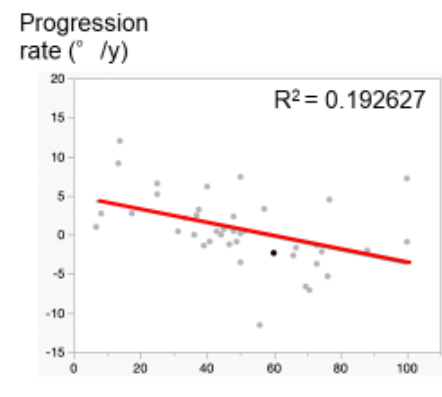

Initial Correction (\%)

Figure 5. Relation of Initial Correction $(\%)$ with Progression Rate $(\% / y)$ (The correlation between the progression rate and the percentage of initial correction by cast application was recognized in all patients $(\mathrm{P}=0.0113)$ and the Cast Mild-Group $(\mathrm{P}=0.0041)$, but not in the Cast Larger-Group $(\mathrm{P}=0.1153)$

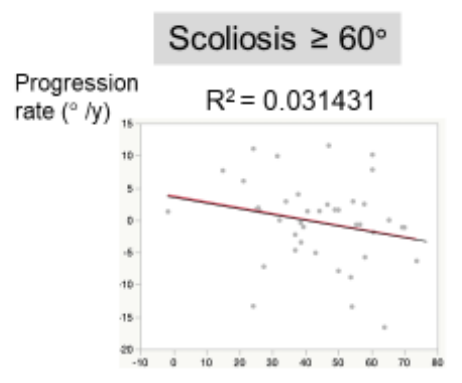

Initial Correction (\%)

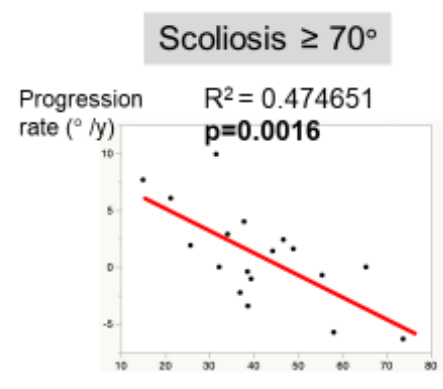

Initial Correction (\%)

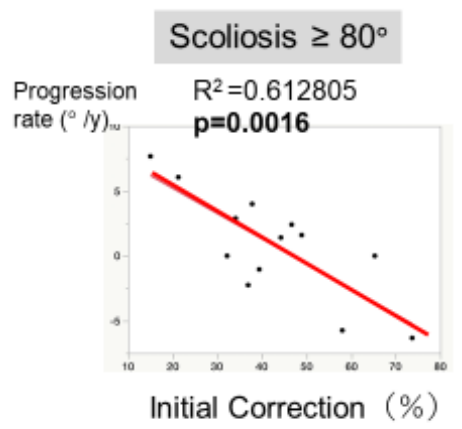

Initial Correction (\%)

Figure 6. Relation of Progression Rate $(\% / y)$ with Initial Correction (\%) by Casting in terms of Magnitude of Scoliosis at the First Cast Placement (All patients were re-categolized into two groups by redefining the curve magnitude for mild and larger groups at three separate intervals $\left(60^{\circ}, 70^{\circ}\right.$, and $\left.80^{\circ}\right)$. The correlation between the progression rate and the percentage of initial correction by cast application became more significant in patients with a higher scoliosis magnitude at the first cast application (the group with scoliosis $\geq 70^{\circ}$ and $\geq 80^{\circ}$ )

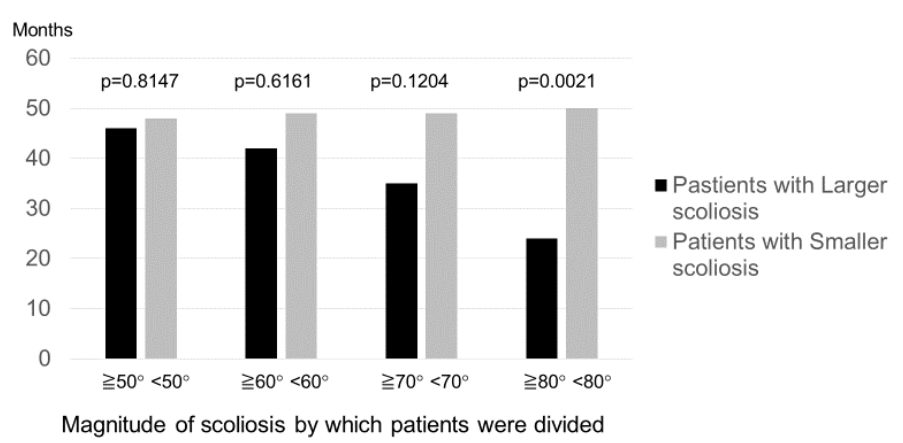

Figure 7. Duration of Delayed Tactic of ASRCB-T for Surgery (In this graph, all patients were divided into two groups; smaller and larger group by initial scoliosis of $50^{\circ} 60^{\circ}, 70^{\circ}$, and $80^{\circ}$ Patients in the larger group whose initial scoliosis was larger than $80^{\circ}$ showed significant shorter duration $(P=0.0021)$ of its ability to delay with only 23 months $)$ 
had Marfan syndrome and funnel chest. All patients had superior mesenteric artery syndrome at the fifth cast placement, resulting in a strategic change to surgical intervention.

\section{Discussion}

Correcting severe scoliosis and suppressing the progression of scoliosis while preserving the growth potential, especially in very young patients with EOS, is highly challenging. In the last two to three decades, several surgical treatment methods have been invented and introduced [16-18]. Nevertheless, recent studies have reported a higher rate of postoperative complications, some of them caused the deterioration of spinal deformity and chest cage deformity [1-5]. Hence, serial cast treatment returned to the clinical practice of EOS. Some studies demonstrated that serial cast treatment works proficiently not only in terms of the reduction of scoliosis in patients with relatively small-magnitude scoliosis but also as a delayed tactic for surgical intervention [6-10].

In the present study, scoliosis just before the first cast application was maintained at almost the same magnitude as that at the end of ARCB-T in both the groups, suggesting that the efficacy of ARCB-T in terms of suppression of scoliosis progression could not be any different in almost all EOS patients without any impact of the scoliosis magnitude at the time of the first cast placement if they are indicated for ARCB-T. Alternatively, the severity of scoliosis did not seem to affect the initial percentage of the correction and progression rate unless a rib cage deromrity such as windswept thoracic cage deformity or severe funnel chest was associated with scoliosis. This chest cage deformity was contraindication for corrective cast application. Overall, it was likely that smaller scoliosis at the start or ARCB-T results in smaller scoliosis at the end of ARCB-T, and larger scoliosis results in larger scoliosis displaying the same progression rate.

The reduction of scoliosis was recognized in some patients with all kinds of etiologies. IIS exhibited the highest percentage of the reduction in all etiologies, irrespective of the magnitude of scoliosis at the start of ARCB-T, as reported previously [7-12]. Regarding the magnitude of scoliosis at the start of serial cast treatment, the present study reported that $70^{\circ}$ was, perhaps the limit of the reduction of scoliosis in EOS. Precisely, patients with $>70$ of scoliosis had virtually no chance to attain the improvement of scoliosis by ARCB-T.

In addition, the present study demonstrated that ARCB-T attained an almost similar progression rate irrespective of the magnitude of scoliosis in EOS patients. Moreover, if the first cast placement attained a better percentage of the correction of scoliosis, the progression rate of scoliosis could be suppressed with a higher probability even in patients with larger scoliosis, suggesting that serial cast treatment like ARCB-T exhibited higher likelihood in the suppression of the scoliosis progression even in patients with much larger scoliosis, such as scoliosis of around $70^{\circ}$ or $80^{\circ}$. Therefore, surgical intervention could be delayed by ARCB-T for some, though not all, patients with much larger scoliosis because of the suppression of the scoliosis progression. Nonetheless, the larger the scoliosis at the first cast application, the harder it is to delay surgical intervention using ARCB-T. According to the results of this study, $70^{\circ}-80^{\circ}$ of scoliosis at the initiation of ARCB-T may be the upper limits for the effectiveness of ARCB-T as an efficient delaying tactic for surgery.

Cast application-related complications for patients with larger scoliosis should be particularly given attention during the first and second days after cast placement. In the present study, we excluded slight pressure sore, such as redness and skin sloughing, from major complications because they did not affect the ARCB-T schedule; however, these complications occurred in almost all patients with larger scoliosis. Hence, we ascertained the duration of cast application for 2-3 weeks in ARCB-T; other reasons included ethnic customs of the Japanese who like taking a bath almost every day, high humidity in Japan, and no anesthetic risk for repeated cast placement. In this study, severe complications during ARCB-T occurred in five patients, all with larger scoliosis. Despite their low frequency, severe complications should be carefully monitored for prompt detection when performing cast placement, especially for patients with larger curvatures or very small children.

The present study has several limitations. First, we compared patients with smaller scoliosis and larger scoliosis to investigate the efficacy of ARCB-T for patients with larger scoliosis in terms of the reduction of scoliosis and delayed tactic for surgery. Thus, it might be more appropriate to compare patients with larger scoliosis who underwent ARCB-T and those who did not undergo ARCB-T. However, it was practically impossible to monitor those patients without any treatment. From the perspective of morality, withholding treatment can never be accepted in clinical practice.

Second, ARCB-T can be considered one of the serial cast treatments. Nevertheless, cast application in ARCB-T is entirely different from those reported previously [6,7]. Comparing the efficacy of serial cast treatment with other different types of serial cast treatment could be challenging. Third, each surgeon has a different strategy for surgical treatment for progressive EOS. In the present study, all decisions of surgical intervention were taken by a senior author (NK) based on the strategy illustrated in the presentation of ARCB-T. It might be challenging to conclude that serial cast treatment could be useful as a delayed tactic even for larger scoliosis from only one doctor's case series.

Finally, the present study enrolled patients with various etiologies. CS/ST were leading in each group. In addition, EOS patients were treated with ARCB-T at a general hospital and not at a children's hospital, where the orthopedic department focuses on spine surgery of both children and adults. As patients with marginal scoliosis are not referred to our institution just for observation, we enrolled a relatively small number of patients with IIS compared with other reports [7-10]. Despite these limitations, it can be determined that ARCB-T, a type of serial cast treatment, could be one of the options for EOS patients with even larger scoliosis. Nevertheless, further investigation is warranted to investigate the efficacy and limitations of serial cast treatment for larger EOS.

\section{Conclusion}

In conclusion, better correction at the first cast application in ARCB-T was a useful indicator to estimate the efficacy of ARCB-T as a delayed tactic for surgery even for patients with larger scoliosis. Although ARCB-T works less efficiently as a delayed tactic to surgery for larger scoliosis, it might be worthwhile applying even for largermagnitude scoliosis if it displays better correction at the first cast placement. Hence, this study suggests that ARCB-T could be one of the options for EOS patients even with larger scoliosis in terms of the improvement of scoliosis and a delayed tactic as observed in relatively smaller curvature in EOS. 


\section{Conflicts of interest}

There are no Conflict of Interest to declare. No benefits in any form have been received from commercial party related directly or indirectly to the subject of this manuscript.

\section{Acknowledgment}

We sincerely express our appreciation to Ms. S Hiraiwa and Ms. N Kojima for their contribution to this project.

\section{Funding}

The study was supported by a grant from the research on Policy Planning and Evaluation of the Ministry of Health Labour and Welfare in Japan.

\section{References}

1. Bess S, Akbarnia BA, Thompson GH (2010) Complications of growing rods. Treatment for early-onset scoliosis: analysis of one hundred and forty patients. J Bone Joint Surg Am 92: 2533-2543. [Crossref]

2. Sankar WN, Skaggs DL, Yazici M (2011) Lengthening of dual growing rods and the law of diminishing returns. Spine 36: 806-809. [Crossref]

3. Yang JS, Sponseller PD, Thompson GH (2011) Growing Spine Study Group. Growing rod fractures: risk factors and opportunities for prevention. Spine 36: 1639-1644. [Crossref]

4. Lucas G, Bollini G, Jouve JL (2013) Complications in pediatric spine surgery using the vertical expandable prosthetic titanium rib-the French experience. Spine 38: E1589-E1599. [Crossref]

5. Watanabe K, Uno K, Suzuki T (2013) Risk factors for complications associated with growing-rod surgery for early-onset scoliosis. Spine 38: E464-E468. [Crossref]

6. Mehta MH (2005) Growth as a corrective force in the early treatment of progressive infantile scoliosis. J Bone Joint Surg Br 87: 1237-1247. [Crossref]
7. Sanders JO, D'Astous J, Fitzgerald M, (2009) Derotational casting for progressive infantile scoliosis. J Pediatr Orthop 29: 581-587.

8. D'Astous JL, Sanders JO (2007) Casting and traction treatment methods for scoliosis Orthop Clin N Am 38: 477-481.

9. Fletcher ND, McClung A, Rathjen KE, Denning JR, Browne, R, et al. (2012) Serial Casting as a Delay Tactic in the Treatment of Moderate-to-Severe Early-onset Scoliosis. $J$ Pediatr Orthop 32: 664-671. [Crossref]

10. Wardron SR, Roe-Kochert C, Sib-Hing JP (2013) Early onset scoliosis: The Value of Serial Risser Casts. J Pedatr Orthop 33: 775-780. [Crossref]

11. Gussous YM, Tarima S, Xhao (2015) Serial Derotational Casting in Idiopathic and Non-Idiopathic Progressive Early-Onset Scoliosis. Spine Deformity 3: 233-238.

12. Iorio J, Orland G, Diefenbach C (2017) Serial Casting for Infantile Idiopathic Scoliosis: Radiographic Outcomes and Factors Associated with Response to Treatment. J Pediatr Orthop 37: 311-316. [Crossref]

13. Demirkiran HG, Bekmez S, Celilov R (2015) Serial Derotational Casting in Congenital Scoliosis as a Time-buying Strategy. J Pediatr Orthop 35: 43-49.

14. Kawakami N, Koumoto I, Dogaki Y (2018) Clinical Impact of Corrective cast Treatment for Early Onset Scoliosis: Is it a Worthwhile Treatment Option to Suppress Scoliosis Progression Before Surgical Intervention? J Pediatr Orthop 38: e556-561.

15. Willians BA, Matsumoto H, McCalla DJ (2014) Development of Initial Validation of the Classification of Early-Onset Scoliosis (C-EOS). J Bone Joint Surg Am 96: 13591367.

16. Campbell RM Jr, Adcox BM, Smith MD (2007) The effect of mid-thoracic VEPTR opening wedge thoracostomy on cervical tilt associated with congenital thoracic scoliosis in patients with thoracic insufficiency syndrome. Spine 32: 21712177.

17. Akbarnia BA, Marks DS, Boachie-Adjei O (2017) Dual growing rod technique for thetreatment of progressive early-onset scoliosis: a multicenter study. Spine 30: S46-S57. [Crossref]

18. Akbarnia BA, Cheung K, Noordeen H (2013) Next generation of growth-sparing technique: preliminary clinical results of a magnetically controlled growing rod (MCGR) in 14 patients with early onset scoliosis. Spine 38: 665-670.

Copyright: $@ 2019$ Kawakami N. This is an open-access article distributed under the terms of the Creative Commons Attribution License, which permits unrestricted use, distribution, and reproduction in any medium, provided the original author and source are credited. 